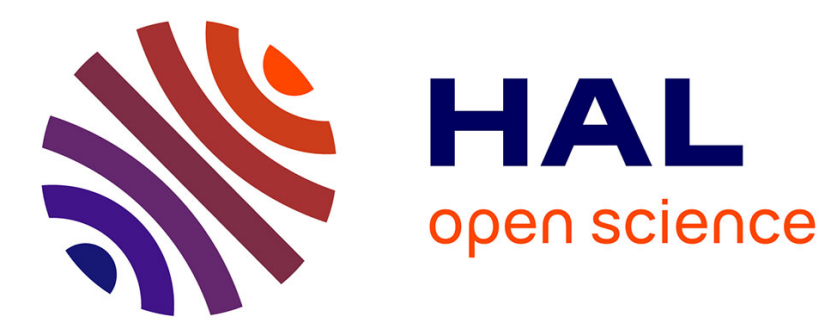

\title{
RT Loop Flexibility Enhances the Specificity of Src Family SH3 Domains for HIV-1 Nef $\dagger, \ddagger$
}

\author{
Stefan Arold, Ronan O'Brien, Peet Franken, Marie-Paule Strub, Francois
}

Hoh, Christian Dumas, John E Ladbury

\section{- To cite this version:}

Stefan Arold, Ronan O'Brien, Peet Franken, Marie-Paule Strub, Francois Hoh, et al.. RT Loop Flexibility Enhances the Specificity of Src Family SH3 Domains for HIV-1 Nef $\dagger$, $\ddagger$. Biochemistry, 1998, 37 (42), pp.14683-14691. 10.1021/bi980989q . hal-02359646

\section{HAL Id: hal-02359646 \\ https://hal.science/hal-02359646}

Submitted on 14 Nov 2019

HAL is a multi-disciplinary open access archive for the deposit and dissemination of scientific research documents, whether they are published or not. The documents may come from teaching and research institutions in France or abroad, or from public or private research centers.
L'archive ouverte pluridisciplinaire HAL, est destinée au dépôt et à la diffusion de documents scientifiques de niveau recherche, publiés ou non, émanant des établissements d'enseignement et de recherche français ou étrangers, des laboratoires publics ou privés. 


\title{
RT Loop Flexibility Enhances the Specificity of Src Family SH3 Domains for HIV-1 Nef ${ }^{\dagger, \ddagger}$
}

\author{
Stefan Arold, ${ }^{\S}$ Ronan O’Brien," Peet Franken, ${ }^{\S}$ Marie-Paule Strub, ${ }^{\S}$ Francois Hoh, ${ }^{\S}$ Christian Dumas, ${ }^{\S}$ and \\ John E. Ladbury*,II \\ Centre de Biochimie Structurale, UMR C9955 CNRS, U414 INSERM, Université Montpellier 1, Faculté de Pharmacie, Avenue \\ C. Flahault, F34060 Montpellier, France, and Department of Biochemistry and Molecular Biology, University College London, \\ The Darwin Building, Gower Street, London, WC1E 6BT, U.K.
}

Received April 30, 1998; Revised Manuscript Received August 14, 1998

\begin{abstract}
Understanding the issue of specificity imposed in the interactions of SH3 domains has largely been addressed in studies investigating the interaction of proline-rich amino acid sequences derived from potential ligands for these domains. Although the interaction with this motif forms an essential platform in the binding of SH3 domains, in many cases little specificity is observed and the difference in affinity for so-called specific and nonspecific proline-rich sequences is not great. Furthermore, the binding interface between an $\mathrm{SH} 3$ domain and a protein ligand appears to encompass more interactions than are represented by that involving the proline-rich motif. Here we investigate the issue of specificity from the opposite point of view; namely, how does a ligand recognize different SH3 domains? We present the crystal structure of the unbound SH3 domain from hemopoietic cell kinase (Hck) which is a member of the Src family of tyrosine kinases. This structure reveals that, unlike the structures of other Src kinase SH3 domains, the RT loop region is highly mobile and lacks a network of hydrogen bonds that is elsewhere apparent. The RT loop has been shown to form a major part of the binding interface between SH3 domains and HIV-1 Nef. Thermodynamic data, derived from isothermal titration calorimetry, for the binding of Hck SH3 to HIV-1 Nef show that the binding of Hck $\left(K_{\mathrm{D}}=1.5 \mu \mathrm{M}\right)$ is approximately an order of magnitude tighter than those of other Src family kinases that were investigated (Fyn, Lck, and $\mathrm{Src})$. This increase in affinity is attributed to, among other effects, the inherent flexibility in the RT loop which does not require breaking the network of hydrogen bonds to adopt the conformation required for binding.
\end{abstract}

The defining interaction for Src homology 3 (SH3) domains was initially deemed to be with that of a polyproline-based sequence on the surface of the ligand $(1,2)$. Structural studies subsequently revealed that the ability of these sequences to adopt a left-handed polyproline type II (PPII) helical conformation was necessary for specific binding (3-9). A plethora of studies attempted to identify how a given $\mathrm{SH} 3$ domain can discriminate between various proline-rich sequences in peptides derived from potential physiological ligands $(5,9,11-15)$. Some SH3 domains do have a weakly delineated preference for some prolinerich sequences. However, since the PPII helix is a common secondary structural element found on the surface of many

This study was supported by funding from the ANRS and FRM Sidaction. S.A. is a Predoctoral Fellow of Ministére de l'Enseignement Supérieur et de la Recherche. J.E.L. is a Wellcome Trust Senior Research Fellow.

$\ddagger$ The structure of the $\mathrm{SH} 3$ domain from Hck has been assigned the Brookhaven Database PDB filename 0000.

* Address correspondence to this author.

$\S$ Université Montpellier 1.

"University College London.

${ }^{1}$ Abbreviations: SH3, Src homology 3; Hck, hemopoietic cell kinase; HIV, human immunodeficiency virus; Nef, negative factor; ITC, isothermal titration calorimetry; GST, glutathione-S-transferase; PPII, polyproline type II helix; RT loop, loop in SH3 structure named after arginine, $\mathrm{R}$, and threonine, $\mathrm{T}$, residues commonly found in it. globular proteins, and the site of interaction of this helix on the SH3 domain is essentially featureless, being formed between highly conserved hydrophobic residues, additional specificity is likely to be derived elsewhere in the biomolecular interface. Binding studies between SH3 domains and proteins suggest that there are significant interactions over and above those represented by the burial of the prolinerich motif $(16,17)$. Recent X-ray crystallographic data on the complex between the SH3 domain from the Src family protein Fyn and the Nef protein of human immunodeficiency virus 1 (HIV-1) $(18,19)$ confirm that there is a large interfacial contact surface (which contains the canonical ProX $x x X x x$ Pro motif; where $\mathrm{Xxx}$ is any amino acid). Thus, although providing a necessary platform, the interaction of the proline-rich sequence with an SH3 domain is not entirely sufficient for dictating specificity.

Here we address the issue of specificity from a different perspective; namely, what are the features of SH3 domains which allow a protein ligand to discriminate between them? For this investigation, we adopt the interaction of HIV-1 Nef with SH3 domains from the Src family. This requires the correlation between structural detail and thermodynamic data for complex formation. We present the crystal structure of the unbound SH3 domain from hemopoietic cell kinase (Hck), a member of the Src family. The structures of the 


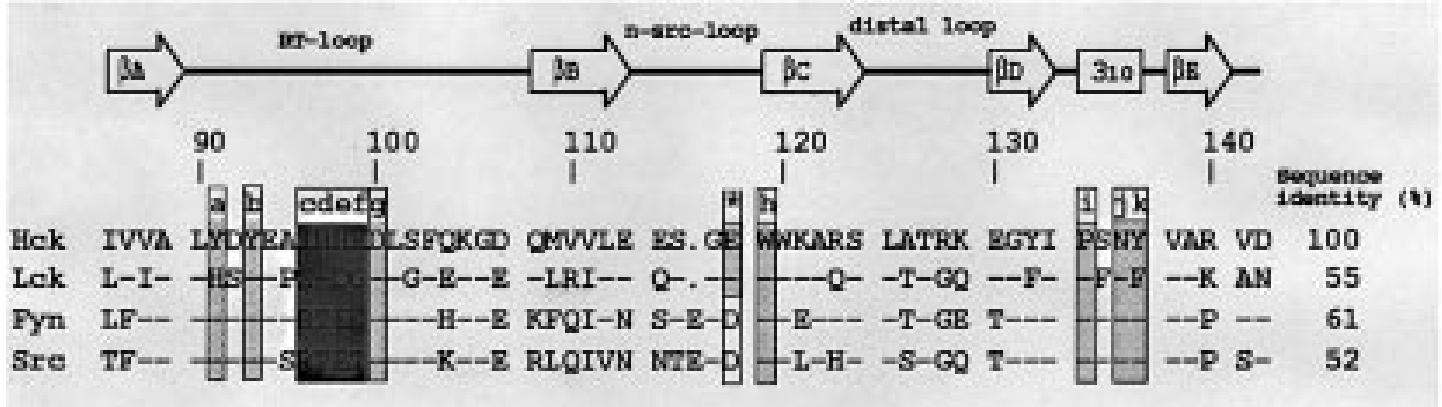

FIGURE 1: Sequence alignment of Src family SH3 domains from Hck, Fyn, Lck, and Src showing regions associated with secondary structural elements common to Src family SH3 domains. The figure also highlights amino acids (a-k) involved in binding to HIV-1 Nef (19). Light gray: nonspecific interactions with the Nef ProXxxXxxPro region of Nef. Dark gray: specific 'tertiary' interactions. Asterisk: Amino acid implicated as possibly binding in modeling studies herein reported.

isolated ligand-free SH3 domain from Fyn (20, 21), Src (22), and Lck (23) have been previously reported. Furthermore, the structures of the full-length 'auto-regulated' or 'closed' form of the tyrosine kinases Src and Hck, in which the SH3 domain forms an intimate interaction with the intramolecular kinase domain, have also been determined $(24,25$, respectively). These structures reveal that, despite the high level of homology between the sequences of these Src family SH3 domains (Figure 1), significant amino acid substitutions occur on the surface which is likely to constitute the Nef interface outside the proline-rich binding site region. Thermodynamic data on the interactions of these SH3 domains in light of this structural information enable definition of the salient features in their recognition by Nef.

The human immunodeficiency virus HIV-1 Nef protein binds with varying affinity to a subset of $\mathrm{SH} 3$ domains from proteins in the Src family of tyrosine kinases [Fyn, Hck, Lck, and $\operatorname{Lyn}(26,16,27,28$, respectively)]. Although this protein has no known catalytic activity, on viral infection it is one of the first to be produced at high levels. Nef is encoded by human and simian immunodeficiency viruses (HIV and SIV) where it is necessary for efficient replication of the virus and the development of the pathology associated with AIDS. Two major effects of Nef on cell function have consistently been described (for reviews, see 29, 30): (1) the induction of alterations in cellular signal transduction pathways; and (2) the down-regulation surface expression of CD4 (primary viral receptor in T-cells), and MHC class 1 molecules $(31,32)$. The correlation between the increase in infectivity and the interaction of Nef with SH3 domains from Src family kinases has been established. Furthermore, direct evidence for the effects of binding to SH3 domains of Src family kinases has been demonstrated. For example, the formation of the Nef-Hck complex, which is necessary and sufficient for the transformation of Rat-2 fibroblasts, was completely blocked by mutagenesis of the Nef proline-rich motif (33). Fyn and Lck are functionally associated with the T-cell antigen receptor (TcR)/CD3 receptor complex and are activated during the course of TcR and CD4 engagement. Nef blocks the early events in TcR/CD3 signaling (34) and is very likely to do so upon association with the Src kinases Lck and Fyn. As Lck is associated with CD4, the binding of Nef to Lck may well play a key role in Nef-mediated down-regulation of CD4 (35-37). There is no direct evidence for the in vivo interaction of HIV-1 Nef with Src; however, association has been reported in the case of a Nef isolate from simian immunodeficiency virus (SIV) (38).
Based on the homology of SH3 domains from the Src family tyrosine kinases, we decided to include this domain in this investigation as a further probe of specificity. The involvement of the Nef-SH3 domain interactions in the infectivity of HIV-1 has made it the focus of a number of drug design efforts, and therefore, understanding the determinants of specificity in its interactions is fundamental to pharmaceutical intervention.

The structural detail and the thermodynamic data reveal the importance of interactions outside those represented by the proline-rich motif interface. Particular importance is attributed to the interaction of the RT loop region from the SH3 domains, and the differences in the thermodynamic parameters of the studied Src family SH3 domains can be attributed (at least in part) to the effect of the inherent flexibility of this loop in binding.

\section{EXPERIMENTAL PROCEDURES}

Preparation of Recombinant Proteins. HIV-1 LAI Nef full length (residues 1-206), $\mathrm{Nef}_{\Delta 1,57}$ (residues 58-206), and the $\mathrm{SH} 3$ domains of Hck (residues 82-142 of full-length Hck), Src (residues 82-142), and Fyn (residues 72-142) were expressed in Escherichia coli as fusion proteins with glutathione-S-transferase (GST) utilizing the pGEX-2T gene fusion vector (Pharmacia). All constructs were transformed into E. coli BL21 cells (Pharmacia), except the Src SH3GST plasmid, that was transformed into $E$. coli DH5 $\alpha$ cells. The Lck SH3 domain (residues 63-120) was cloned as a GST fusion protein in the pGEX $3 X$ vector containing a factor Xa cleavage site and expressed in E. coli DH5 $\alpha$ cells. Cells grown overnight at $37{ }^{\circ} \mathrm{C}$ in Luria-Bertani medium containing $1 \mathrm{mg} / \mathrm{mL}$ ampicillin were diluted 1:50 into fresh medium and grown at $37{ }^{\circ} \mathrm{C}$ for $3-4 \mathrm{~h}$ until an optical density of 0.6 at $\lambda=600 \mathrm{~nm}$. Isopropyl $\beta$-D-thiogalactopyranoside was then added to $0.2 \mathrm{mM}$, and the cells were grown for an additional 2-3 h. Cells were harvested by centrifugation ( $3000 \mathrm{~g}$ for $30 \mathrm{~min}$ ) and stored at $-80{ }^{\circ} \mathrm{C}$. For purification, the frozen cells were resuspended in 10 volumes of ice-cold resuspension buffer [PBS buffer (GibcoBRL), $2.5 \mathrm{mM}$ EGTA (BDH Biochemical), $5 \mathrm{mM}$ benzamidine (Sigma), and in the case of Nef, $5 \mathrm{mM}$ dithiothreitol (BDH Biochemical)]. Prior to mild sonication on ice $(4 \times 20 \mathrm{~s})$, $1 \%$ Triton and $5 \mathrm{mM}$ phenylmethylsulfonyl fluoride (PMSF, Sigma) were added. The lysed cells were centrifuged $(50000 \mathrm{~g}$ for $1.5 \mathrm{~h})$, and the pellet was discarded. The supernatant was loaded onto a glutathione-Sepharose 4B 
column (Pharmacia) and equilibrated in resuspension buffer. The beads were then extensively washed with 100 bed volumes of $1 \mathrm{M} \mathrm{NaCl}$ in resuspension buffer to eliminate nonspecifically fixed products, and equilibrated in the cleavage buffer (50 mM Tris-HCl, pH 7.5, $150 \mathrm{mM} \mathrm{NaCl}$, $2 \mathrm{mM} \mathrm{CaCl}_{2}$, and $5 \mathrm{mM}$ DTT in the case of Nef). The proteins were cleaved from the fused GST protein on the column matrix by overnight treatment at room temperature with $7-10$ units $\cdot \mathrm{mL}^{-1}$ bovine thrombin (CalBiochem) (Nef, Hck, Src, and Fyn) or $10 \mu \mathrm{g}$ of factor Xa (Pharmacia) (Lck) per milligram of fusion protein. The thrombin and factor Xa cleavage was stopped with $5 \mathrm{mM}$ benzamidine and 5 mM DTT, respectively. The cleaved proteins were eluted and their purity confirmed by SDS-PAGE. Proteins that were readily $>95 \%$ pure (Lck SH3, $\mathrm{Src} \mathrm{SH} 3$, and $\mathrm{Nef}_{\text {full length }}$ ) were concentrated using $3 \mathrm{kDa}$ cutoff ultrafiltration (Amicon) and dialyzed against the titration buffer $[20 \mathrm{mM}$ phosphate buffer (BDH Biochemical), pH 7.5, $150 \mathrm{mM} \mathrm{NaCl}, 2 \mathrm{mM}$ EGTA, and $5 \mathrm{mM}$ DTT]. Proteins showing contaminants $\left(\mathrm{Nef}_{\Delta 1,57}, \mathrm{Hck}\right.$, and Fyn SH3) were further purified using size exclusion chromatography (Pharmacia S-100 HR). Fractions containing $>95 \%$ pure protein were pooled, concentrated, and dialyzed against the titration buffer for thermodynamic analysis. For crystallization experiments, the Hck SH3 domain was dialyzed against $20 \mathrm{mM}$ Tris- $\mathrm{HCl}$, $\mathrm{pH} 8.0,100 \mathrm{mM} \mathrm{NaCl}$, and $1 \mathrm{mM}$ EGTA and concentrated by ultrafiltration up to $6 \mathrm{mg} / \mathrm{mL}$.

The integrity of the purified proteins was checked by mass spectroscopy and N-terminal sequencing. Protein concentrations were estimated by UV absorption of aromatic residues at $280 \mathrm{~nm}$ using the following extinction coefficients: Hck, Src, Fyn SH3 domains, $\epsilon_{280 \mathrm{~nm}}=16500 \mathrm{M}^{-1} \mathrm{~cm}^{-1}$; Lck SH3 domain, $\epsilon_{280 \mathrm{~nm}}=12660 \mathrm{M}^{-1} \mathrm{~cm}^{-1}$; full length Nef, $\epsilon_{280 \mathrm{~nm}}$ $=49150 \mathrm{M}^{-1} \mathrm{~cm}^{-1}$; and $\mathrm{Nef}_{\Delta 1-57}, \epsilon_{280 \mathrm{~nm}}=31960 \mathrm{M}^{-1}$ $\mathrm{cm}^{-1}$.

Crystallization. Crystals of the Hck SH3 domain were grown by vapor diffusion with the hanging drop technique at $21{ }^{\circ} \mathrm{C}$. For crystal growth, $1 \mathrm{cmc}$ of octyl $\beta$-glucoside was added to the protein solution containing the purified Hck SH3 domain at a concentration of $4.3 \mathrm{mg} / \mathrm{mL}$ in its storage buffer $(20 \mathrm{mM}$ Tris- $\mathrm{HCl}, \mathrm{pH} 8.0,100 \mathrm{mM} \mathrm{NaCl}$, and $1 \mathrm{mM}$ EGTA). For one crystallization experiment, $2 \mu \mathrm{L}$ of this protein solution was mixed with an equal volume of the well solution [100 mM bicine, $\mathrm{pH}$ 9.3, 3.7 M sodium formate, 2\% poly(ethylene glycol) (PEG 3000)]. Crystals grew reproducibly over a period of 1 week with final dimensions of $(350 \times 350 \times 100) \mu \mathrm{m}$ in the best cases. Hck SH3 crystals belong to space group $P 22_{1} 2{ }_{1}$ with cell dimensions of $a=51.50 \AA, b=106.15 \AA$, and $c=78.80 \AA$. Six independent Hck SH3 molecules were present in the asymmetric unit, resulting in a Matthews coefficient of $2.6 \AA^{3} /$ Da.

Data Collection. Diffraction data up to $2.6 \AA$ resolution were recorded at $1.074 \AA$ at the ESRF synchrotron light source (beamline D2AM) in Grenoble, France, on two different crystals grown under identical conditions. Data from both crystals were processed with an adapted version of the XDS program (39) and merged to finally obtain a 93\% complete data set $(62 \%$ for the last resolution shell between 2.6 and $2.72 \AA$ spacings) containing 13043 unique reflections between 34 and $2.6 \AA$ resolution.
Structure Determination and Refinement. The structure was determined by molecular replacement with the AMoRe program (40) using the Hck SH3 domain from the published Hck kinase (PDB entry 2HCK, 25) as molecular template. Five of the expected six molecules could be placed by AMoRe. The last molecule was retrieved by iterative phase improvment using a partial model corresponding to the five SH3 molecules and a molecular envelope covering all six molecules. The missing SH3 molecule was clearly identifiable in the resulting $2 F_{\mathrm{o}}-F_{\mathrm{c}}$ map and was unambiguously fitted into this density.

Model refinement was carried out using X-PLOR (41). Bulk-solvent corrections and anisotropic temperature factor scaling were applied during refinement. Program 'O' (42) was used for visualization and model building, PROCHECK (43) and WHAT IF (44) for displaying the quality of the model. All recorded data from 34 to $2.6 \AA$ were used in refinement. All loop regions were rebuilt into the simulated annealing omit maps calculated by X-PLOR. The final model comprises 6 Hck SH3 molecules $(\mathrm{A}-\mathrm{F}$, including residues 84-140 following the conventions used for the Hck kinase) and 75 water molecules. The final model shows good stereochemistry: the rms deviations for ideal bond distances and bond angles are $0.010 \AA$ and $1.27^{\circ}$, respectively. Main chain $\phi$ and $\psi$ torsion angles for $90 \%$ of the non-glycine residues fall within the most-favored region of the Ramachandran plot. The final crystallographic $R$ factor for all reflections between 34 and $2.6 \AA$ resolution is $23.3 \%$, and the $R_{\text {free }}$ value is $29.7 \%$. The overall temperature factors for the six SH3 molecules are 18.4, 21.5, 22.5, 27.7, 28.6, and $29.7 \AA^{2}$, respectively.

Isothermal Titration Calorimetric (ITC) Experiments. Titration experiments were carried out with an MCS calorimeter (MicroCal Inc., Northampton, MA) (45, 46), and performed as described in (47). Before titrations, both reactants were dialyzed exhaustively, in the same container, against the titration buffer: $20 \mathrm{mM}$ phosphate buffer, $\mathrm{pH}$ $7.5,150 \mathrm{mM} \mathrm{NaCl}, 2 \mathrm{mM}$ EGTA, and $5 \mathrm{mM}$ DTT. Just prior to a titration experiment, the solutions for the syringe and for the cell were thoroughly degassed by stirring under vacuum. Protein concentrations were determined spectrophotometrically by measuring their absorbance at a wavelength of $280 \mathrm{~nm}$. For all reactions, the concentration of the reactant in the syringe was about 10 times greater than in the ITC reaction cell. The protein solutions were injected from a $250 \mu \mathrm{L}$ capacity syringe into the $1.3 \mathrm{~mL}$ calorimeter cell containing the reactant solution. A titration consisted typically of 16-26 injections of 12 or $15 \mu \mathrm{L}$ of SH3 domain (200-600 $\mu \mathrm{M})$, respectively, into the isothermal cell containing Nef protein $(20-60 \mu \mathrm{m})$ at a temperature of $25^{\circ} \mathrm{C}$. Five minutes were allowed between injections for equilibration.

Experimental data were fit using the nonlinear leastsquares algorithm (45) utilizing ORIGIN software (MicroCal Inc.). The heats of dilution for interaction were determined in separate titration experiments by injecting protein solution from the syringe into the appropriate buffer solution in the ITC reaction cell. The heats determined from these experiments were used to correct the titration data prior to data analysis. There was no evidence of associated states of any of the interacting components as assessed by heats of dilution experiments. 


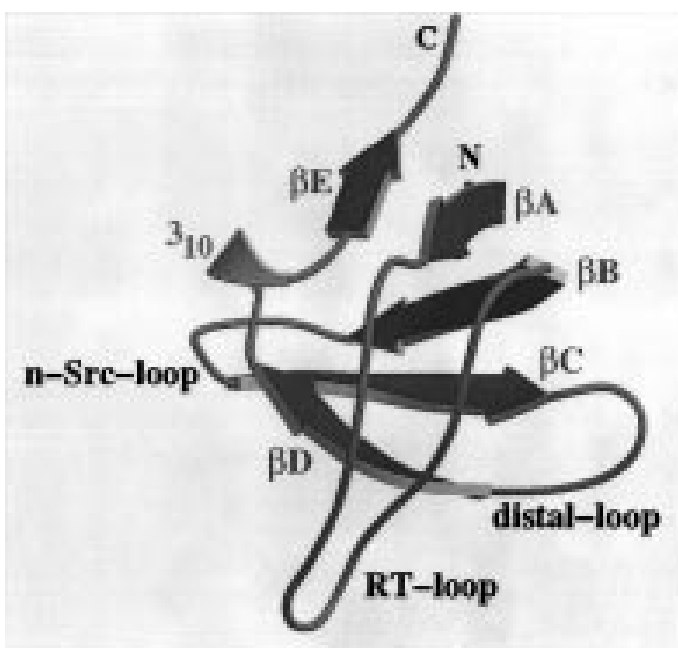

FIGURE 2: Ribbon diagram of Hck SH3 domain showing essential structural features.

Homology Modeling. The crystal structures of HIV-1 $\mathrm{Nef}_{\text {core }}$ complexed with the Ile96Arg and wild-type Fyn SH3 domain (PDB entries 1EFN and 1AVZ) were used as molecular template for modeling of the complexes between $\mathrm{Nef}_{\text {core }}$ and Hck, Lck, and Src SH3 domains. The optimal set of rotamers for the mutated residues was established by cluster analysis of an energy matrix as implemented in the SMD program (48) The modeled complexes containing the new rotamers were then minimized by AMBER (49) in a vacuum using a distance-dependent dielectric constant of $\epsilon$ $=r$. Minimization was carried out under various constraints that were gradually released. The same protocol applied to the original crystal structures yielded modeled structures that were very close to the initial coordinates (rms deviation for all non-hydrogen atoms of 0.7 and $0.8 \AA$ for $1 \mathrm{EFN}$ and 2AVZ, respectively).

\section{RESULTS AND DISCUSSION}

General Features of the Structure of the SH3 Domain from Hck. The Hck SH3 domain forms crystals with six independent molecules in the asymmetric unit related by improper symmetry (see Experimental Procedures). The overall fold of the Hck SH3 domains is the same as reported for the isolated unbound Fyn SH3 domain (20) and for the Hck domain in the context of the whole kinase in the 'closed' conformation (25): five antiparallel $\beta$-strands fold into a $\beta$-barrel of two orthogonal $\beta$-sheets connected by three loops (the RT loop, the nSrc loop, and the distal loop) as shown in Figure 2. A short $3_{10}$ helix is located near the C-terminal (residues 134-137). The $\mathrm{C} \alpha$ trace of the six independent SH3 molecules of the asymmetric unit can be superimposed within an rmsd of $0.47 \AA$, and the $\mathrm{C} \alpha$ traces of the free Hck SH3 structures deviate from the mean Hck SH3 domain bound to the $\mathrm{SH} 2$ domain-kinase domain linker sequence structure (PDB entry 2HCK; 25) by an rmsd of $0.7 \AA$. Perhaps the most noticeable feature of the Hck SH3 structures of the asymmetric unit are the differences in the backbone conformation in the loop regions (Figure 3A). Comparison of the six Hck SH3 domain molecules showed that the largest differences in the $\mathrm{C} \alpha$ coordinates from the mean structure were found for histidine 98 of the RT loop $(0.42 \AA)$, for alanine 126 of the distal loop $(0.86 \AA)$, and for serine 115 from the n-Src loop $(0.40 \AA)$. Within the six independent
A

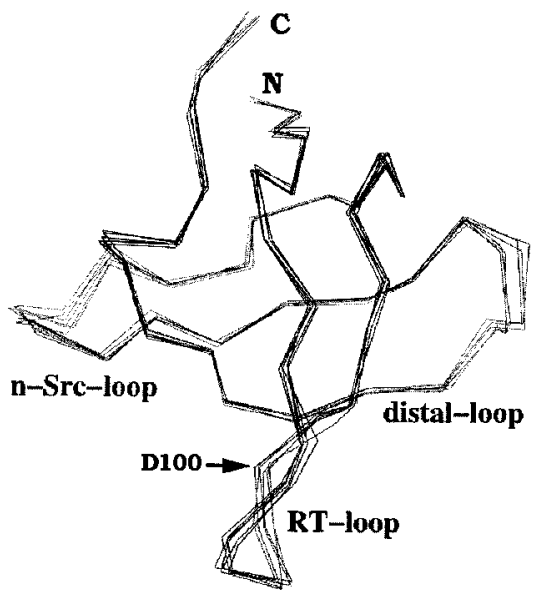

$\mathbf{B}$
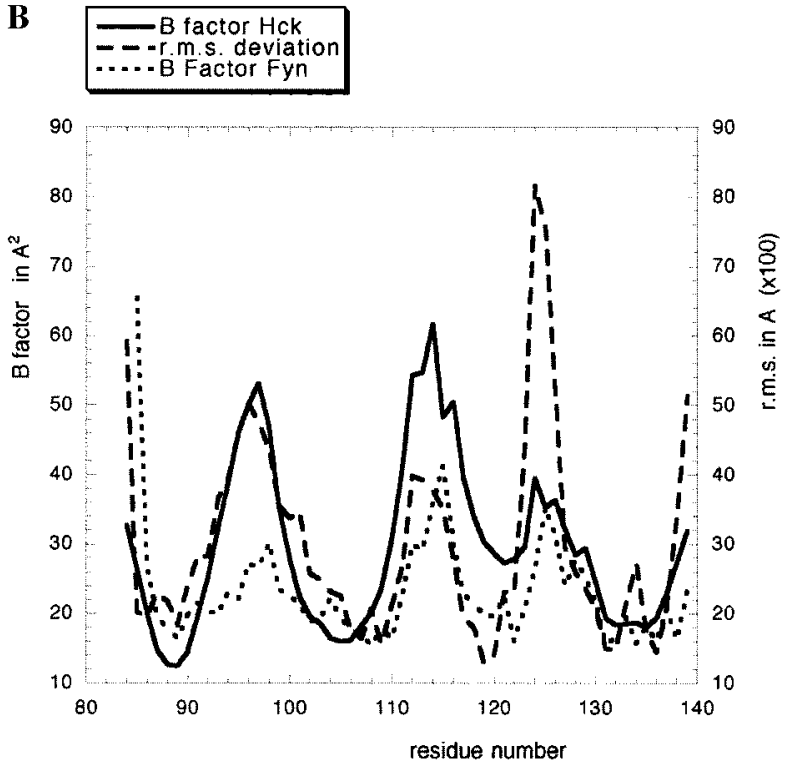

FIGURE 3: Incongruency of the RT loop region of the SH3 domain from Hck. (A) Superposition of the six crystal structure forms of Hck showing loop flexibility (slightly different orientation to that shown in Figure 2). The line corresponds to a trace of the main chain $\alpha \mathrm{C}$ atoms from the crystal structure. (B) Graphical representation of the difference in temperature (B) factors between the SH3 domains from (solid line) Hck and (dotted line) Fyn (20) and the root-mean-square deviation of a single Hck SH3 structure $(\alpha \mathrm{C}$ trace; dashed line) from the mean structure from the six crystal forms in the asymmetric unit. Both figures emphasize the relative disorder in the RT loop region.

structures, many side chains of the loop regions were found in different conformations, and often residual density was observed next to side chain positions, suggesting the possibility of alternative conformations.

Incongruence of RT Loop Conformations between Src Family SH3 Domains. The RT loop region (residues 90108) plays a key role in the binding interface in the structure of the Fyn SH3-Nef complexes $(18,19)$. There is considerable variation in the residues present in the sequences of the RT loops of Src family SH3 domains (Figure 1), suggesting that this region could be important in dictating specificity (16). Comparing structural data on the RT loops of the unbound SH3 domains of Hck and Fyn, in the latter this 19 residue region incorporates an intricate network of hydrogen bonds in which Asp100 plays a central role. This residue potentially forms five hydrogen bonds via its carboxylate group with the side chains of Tyr93 and Thr97 as well as 


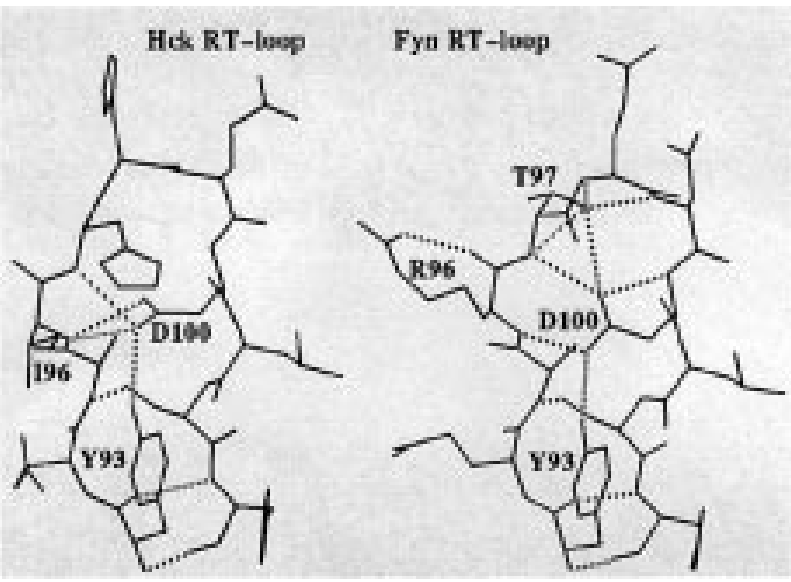

FIGURE 4: Difference between the RT loop regions of Hck and Fyn showing hydrogen bonding conformations. Note the reduction in the extent of the restrictive network of hydrogen bonds in the Hck which is apparent in the Fyn structure centered around Thr97, Arg96, and Asp100.

with the main chains of Thr97 and Arg96. Additional hydrogen bonds are apparent between the side chain of Arg96 and the proximal main chain of Tyr132 of the underlying $\beta$ D-strand. Although Asp100 is conserved in the Hck SH3 domain (as it is in most $\mathrm{SH} 3$ domains), two substitutions (Arg96Ile, Thr97His) preclude the hydrogen bonding network seen in Fyn from forming (Figure 4). This is reflected in the increased flexibility of the RT loop of Hck observed in our structural data described above. Indeed, in the crystal structure of Hck, the two histidines (His97 and -98) in the RT loop are so highly mobile in the unbound state that electron density is often badly defined for the main chain (molecules C, E, and F) and partly or totally absent for most of the side chains (molecules B, C, E, and F). As mentioned above, our crystal structure reveals considerable differences in conformation of the RT loops of the six molecules in the asymmetric unit. The average main chain atom temperature factors for these RT loops (Figure 3B) are $38 \AA^{2}$ (compared to the main chain average of $17 \AA^{2}$ ) whereas in the Fyn SH3 domain this value is significantly lower $\left(25 \AA^{2}, 20\right)$. Both Arg96 and Tyr97 are conserved in the Src SH3 domain, resulting in restriction of mobility of the RT loop observed in Fyn. In Lck, substitution of Arg96Ser and Tyr97His could result in the highly flexible RT loop as observed in Hck; however, the presence of Pro95 is likely to significantly reduce the mobility in this region.

In two of the six Hck molecules (molecules A and D), the Asp100 deviates from its position in the center of the RT loop compared to the other four molecules and to the structure of the whole kinase (25). In a structural superposition of Asp100 in Hck molecules A and C, the orientation is the same as that in the Fyn SH3 domain in complex with Nef. In this complex, Fyn Asp100 bends away from its central position in the RT loop and forms a salt bridge with Arg77 in Nef. The flipping of this Asp results in the aforementioned hydrogen bonding network being broken, resulting in an increase in flexibilty of the loop to interact with the rest of the Nef surface (19). The RT loop is able to adopt a structure which affords a number of hydrogen bonding interactions with Nef. The A and D crystal forms emphasize the strong propensity of Asp100 to form a salt bridging interaction; however, interestingly, this residue is
Table 1: Isothermal Titration Calorimetric Data for the Binding of HIV-1 Nef to SH3 Domains from Src Family Tyrosine Kinases at $25^{\circ} \mathrm{C}$

\begin{tabular}{llrccc}
\hline & & $\begin{array}{c}K_{\mathrm{D}} \\
(\mu \mathrm{M})\end{array}$ & $\begin{array}{c}\Delta G \\
\left(\mathrm{~kJ} \cdot \mathrm{mol}^{-1}\right)\end{array}$ & $\begin{array}{c}\Delta H \\
\left(\mathrm{~kJ} \cdot \mathrm{mol}^{-1}\right)\end{array}$ & $\begin{array}{c}T \Delta S \\
\left(\mathrm{~kJ} \cdot \mathrm{mol}^{-1}\right)\end{array}$ \\
\hline $\mathrm{Nef}_{\Delta 1-57}$ & Hck & 1.5 & -33.3 & -32.6 & 0.7 \\
$\mathrm{Nef}_{\Delta 1-57}$ & Fyn & 15.8 & -27.4 & -2.2 & 25.2 \\
$\mathrm{Nef}_{\Delta 1-57}$ & Lck & 10.6 & -28.4 & -7.0 & 21.4 \\
$\mathrm{Nef}_{\Delta 1-57}$ & Src & 14.3 & -27.6 & -16.2 & 11.4 \\
$\mathrm{Nef}_{\Delta 1-57^{b}}{ }^{b}$ & Hck & 1.5 & -33.3 & -21.6 & 11.7 \\
$\mathrm{Nef}_{\Delta 1-57^{b}}$ & Src & 8.3 & -28.9 & -13.1 & 15.8 \\
$\mathrm{Nef}_{\text {full length }}$ & Hck & 0.6 & -35.5 & -62.8 & -27.3 \\
Nef full length & Src & 11.4 & -28.2 & -41.8 & -13.6 \\
\hline
\end{tabular}

${ }^{a}$ Reported data are based on the means of two individual ITC experiments. All the stoichiometries of the interactions reported are $1.0 \pm 0.2$ based on nonlinear least-squares fitting of the ITC data (45). For conditions for ITC experiments, see Experimental Procedures. ${ }^{b}$ In buffer containing $0.5 \mathrm{M} \mathrm{NaCl}$.

unable to form such a bond in the intramolecular interactions of the SH3 domains in the 'closed' full-length Src and Hck structures $(24,25)$. Thus, Asp100 salt bridge formation appears to be a signature of a specific interaction.

Thermodynamics of Binding of $\mathrm{Nef}_{\Delta l-57}$ to Src Family SH3 Domains. To understand the determinants of specificity, the structural detail from the X-ray crystal structure described herein, and from those previously reported, is correlated to thermodynamic data derived from ITC. Since all the currently reported structural information on $\mathrm{Nef}-\mathrm{SH} 3 \mathrm{com}$ plexes is based on deleted forms of the protein $[\Delta 1-55,(18)$; $\Delta 1-57,(19)]$, we initially report binding data on the deleted form first described by Arold et al. (19). The unliganded $\mathrm{Nef}_{\Delta 1-57}$ construct shows disorder in the $\mathrm{N}$-terminal region from residues 58-73 which includes part of the polyproline region [residues $71-77 ;(19,50)]$. In the bound form, residues $71-77$ adopt the PPII helical conformation with the second half of the polyproline region (74-77) anchored through hydrogen bonding to the Nef core domain (residues 118-121). The dissociation constants for the interactions between $\mathrm{Nef}_{\Delta 1-57}$ and the SH3 domains from Hck, Fyn, Lck, and $\mathrm{Src}$ are all within an order of magnitude of one another (Table 1). Clearly, there is only limited specificity between these interactions; however, within this subset of Src family ligands, Hck binds significantly more tightly than the others. The physiological relevance of this higher affinity is not clear. Fyn, Lck, and Src tyrosine kinases are widely expressed, whereas Hck expression is restricted to hematopoietic cells from the monocyte/macrophage lineage. Thus, in the absence of $\mathrm{Hck}$ in $\mathrm{CD}^{+}{ }^{+} \mathrm{T}$-cells and assuming that the SH3 domain interaction is the only determinant, Nef targets other Src family kinases but with little apparent preference for binding.

The thermodynamic data reveal that for all the SH3-Nef interactions at $25{ }^{\circ} \mathrm{C}$ both the $\Delta H$ and $T \Delta S$ provide a favorable contribution to $\Delta G$ (Table 1). However, in the case of Hck, the $\Delta H$ is the most favorable $\left(-32.6 \mathrm{~kJ} \cdot \mathrm{mol}^{-1}\right)$, and the $T \Delta S$ is the least favorable $\left(0.7 \mathrm{~kJ} \cdot \mathrm{mol}^{-1}\right)$. To analyze the detail of binding specificity, we divide our discussion of these into several independent components. (1) The burial of surface area. The overall favorable entropic contribution to binding in all cases is likely to arise from the dominant effect of the burial of hydrophobic surface area, since on forming the complex water molecules which were ordered 
Table 2: Surface Area ${ }^{a}$ Burial on Formation of Complexes between HIV-1 Nef and SH3 Domains from Src Family Kinases

\begin{tabular}{lcccccccc}
\hline & total & apolar $(\%$ of total) & SH3 & Nef & PPII-SH3 (\% of total) & surface $^{b}$ & surface $^{c}$ & surface $^{d}$ \\
\hline X 1EFN & 1205 & $722(60)$ & 604 & 596 & $714(60)$ & 293 & 262 & 198 \\
M 1EFN & 1288 & $736(57)$ & 638 & 650 & $761(59)$ & 297 & 273 \\
X 1AVZ & 1278 & $684(53)$ & 637 & 641 & $700(55)$ & 253 & 314 \\
M 1AVZ & 1282 & $735(57)$ & 612 & 680 & $674(52)$ & 300 & 303 \\
c-Src 1EFN & 1275 & $713(56)$ & 619 & 656 & $777(61)$ & 265 & 248 \\
c-Src 1AVZ & 1275 & $705(55)$ & 600 & 675 & $679(53)$ & 270 & 289 \\
Hck 1EFN & 1474 & $811(55)$ & 728 & 746 & $827(56)$ & 353 & 330 \\
Lck 1EFN & 1396 & $720(52)$ & 700 & 696 & $798(57)$ & 296 & 330 \\
\hline
\end{tabular}

${ }^{a}$ All areas are given in $\AA^{2}$. Solvent accessibilities were calculated with a rolling sphere of radius of $1.4 \AA$. The modeling protocol leads to a slight overestimation of the buried surface area of about $2-5 \%$. To show bias introduced by the modeling procedure, the minimized PDB coordinates have been included. To show model bias, the complex between HIV-1 Nef and c-Src SH3 is calculated twice, once modeled on the PDB entry

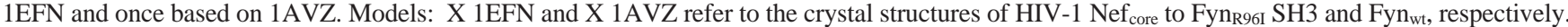
M 1EFN and M 1AVZ designate the minimized PBD entries. c-Src 1EFN and c-Src 1AVZ refer to the c-Src SH3-Nef complex modeled on the basis of PDB entries 1EFN and 1AVZ, respectively. Surface areas: total $=$ total buried surface area $(\mathrm{Nef}+\mathrm{SH} 3)$; apolar $(\%$ of total $)=$ buried hydrophobic atoms, which make X\% of "total"; SH3 = buried surface on the SH3 domain; Nef = buried surface on Nef; PPII-SH3 (\% of total) $=$ buried surface between the PxxP region and the adjacent SH3 residues, making $X \%$ of "total" $(100-X=\%$ of buried surface area for tertiary, non-PxxP-mediated interactions). ${ }^{b}$ Hydrophobic surface within buried surface area for tertiary, non-PxxP-mediated interactions. ${ }^{c}$ Buried surface belonging to SH3 residues contacting tertiary, non-PxxP areas on Nef. ${ }^{d}$ Hydrophobic surface within surface. ${ }^{c}$

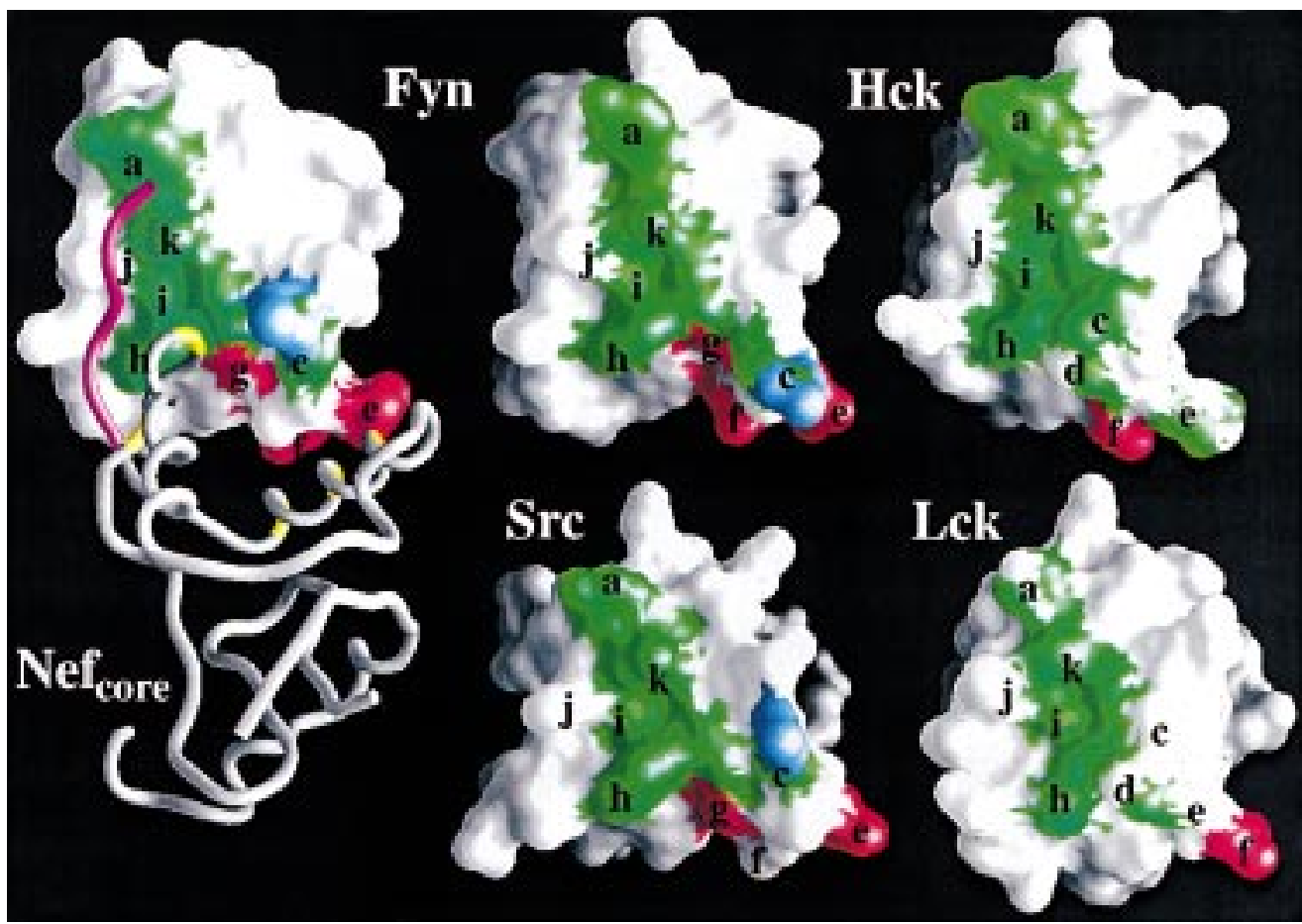

FIGURE 5: Binding surface of Src family SH3 domains showing difference between charged/polar (positive $=$ blue and negative $=$ red) and hydrophobic residues (green). The lower case lettering corresponds to residues involved in binding (see Figure 1). The figure on the left

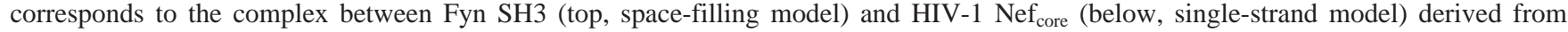
Arold et al. (19). This shows the essential sites of interaction. The PPII helix of Nef (purple) is shown interacting with the hydrophobic surface on the SH3 domain. Also shown are the structures of the unbound SH3 domains from Fyn, Hck, Src, and Lck. The unbound SH3 structures emphasize the similarity of the binding sites presented to interact with HIV-1 Nef.

on this surface are released into the bulk solvent. Table 2 confirms that a large amount of apolar surface is, indeed, buried in all the interactions. This seems to be a common feature associated with SH3 domain binding as was hypothesized in the interaction of Fyn with the p85 subunit of PI3kinase (17). It is worth noting that the interaction of the Hck SH3 domain with Nef involves burial of the largest

${ }^{2}$ The structures described by the PDB filenames in Table 2 are as follows: X 1EFN and X 1AVZ refer to the crystal structures of HIV-1 $\mathrm{Nef}_{\text {core }}$ to $\mathrm{Fyn}_{\mathrm{R} 96 \mathrm{I}} \mathrm{SH} 3$ and $\mathrm{Fyn}_{\mathrm{wt}}$, respectively. M 1EFN and M 1AVZ designate the minimized PBD entries. c-Src 1EFN and c-Src 1AVZ refer to the c-Src SH3-Nef complex modeled on the basis of the PDB entries $1 \mathrm{EFN}$ and $1 \mathrm{AVZ}$, respectively. surface area which would be accompanied by the largest number of released water molecules and hence, in the absence of any other effects, providing the largest favorable entropic contribution of all the interactions. This event is not manifested in the thermodynamic data (Table 1). There is no apparent trend between the burial of surface area and the $K_{\mathrm{D}}$ for the other Src family SH3 domains. (2) The SH3Nef PPII helix interaction. The residues in the Src family SH3 domain interactions with the Nef PPII helix are highly conserved (see Figure 1); thus, this region offers little in the way of specificity. Table 2 and Figure 5 indicate the importance of this part of the interface in providing a large hydrophobic surface for interaction. However, the SH3 
interactions involve a significant amount (almost 50\%) of surface area burial which is derived from outside interactions made with the PPII helix (Table 2). (3) The RT loop interaction. The different residues seen in the RT loop appear to be able to offer some level of specificity. The prime example of this is that Ile96 in the Hck SH3 domain structure is undoubtedly able to probe into a deep pocket on the surface of Nef [as can be ascertained from the Arg96Ile mutation in Fyn $(16,18)$ ]; however, it is not clear that this should result in a much stronger interaction than that associated with the Arg96 residue which is present in the Fyn and Src domains. Furthermore, the interaction of Hck SH3 is likely to be greatly affected by the observation that in the complex interface with Ile96 a water molecule is incorporated (19) whereas in Fyn, where Arg96 occupies the same deep pocket, the water is not present (18). The restriction of this water molecule into the binding interface involves an entropic cost. It is not possible to determine whether the enthalpic gain from hydrogen bonding of this water at the base of the pocket gives rise to an overall favorable $\Delta G(51)$. In the case of the RT loop from Lck, the presence of the hydroxyl group on Ser96 is believed to interact unfavorably with the hydrophobic region of the deep pocket found on the Nef binding site, hence reducing the affinity despite the expected flexibility in the RT loop found in the absence of Arg96 and Thr97 (as discussed below). Figure 5 suggests that one major difference in the binding sites offered to the Nef protein by the SH3 domains is in position 98 where Hck has a His residue whereas Fyn, Src, and Lck have acidic side chains; however, previously reported mutation studies (16) suggest that this is not the case.

The interactions of individual residues, therefore, are unlikely to be solely responsible for the difference in affinity between Hck and the other Src family SH3 domains binding to Nef. The major differences between the $\Delta H$ and $T \Delta S$ terms for Hck compared to the others appear to reflect the increased flexibility of the RT loop region in the former (assuming that Nef does not undergo any significant structural changes between the various SH3 complexes). Part of the interaction of the $\mathrm{SH} 3$ domains requires that the RT loop, in forming its intimate interaction with Nef, assumes a tightly bound rigid conformation in which noncovalent bonds are formed (negative $\Delta H$ ) and conformational entropy is lost (reduced $T \Delta S$ ). As opposed to Hck, in the other SH3 domain interactions (Fyn and Src) the RT loop in the unbound form is restricted by hydrogen bonds which have to be broken before the RT loop can form the required conformation in the bimolecular interface. This requirement to release the RT loop from its more restricted conformation in the unbound form results in a less favorable $\Delta H$ (compared to Hck) due to breaking of hydrogen bonds (see Table 1). On the other hand, the inherent flexibility of the Hck RT ensures that a larger entropic penalty is incurred on binding, whereas in the other SH3 domains going from one restricted conformation (in the unbound form) to another (in the complex) requires less of a penalty and hence the more favorable net $T \Delta S$ values observed.

The affinity of Nef for Fyn, Lck, and Src is remarkably similar; however, the thermodynamic parameters that contribute to the free energy reveal that the interactions are somewhat different. This is emphasized in the difference in the $\Delta H$ and $T \Delta S$ values for the Fyn and Src SH3 domains despite their having a high level of consistency in residues implicated in the binding site with Nef (Figure 1). Indeed, all but one (Thr99) of the amino acid sequence variations between Fyn and Src occur outside the presumed binding site for Nef. This suggests that perhaps the overall stability of the SH3 domain structure is important in dictating the thermodynamics of binding as has been previously inferred for Hck (52).

Effect of Salt Bridge Formation on Binding. The structures of the complexes show that a salt bridge forms between Asp100 of the Fyn SH3 domain and Arg77 of Nef. Both Asp100 and Arg77 are highly conserved in SH3 domains and Nef, respectively. Arg77 plays a key role in the polyproline region and is anchored to the Nef structure through a series of hydrogen bonds locking residues 74-77 into a PPII helix conformation even in the absence of ligand. This salt bridge is located within the center of the interface, and the crystal structure shows clearly defined electron density for both contributing residues (19). Additional charge-charge interactions flanking the binding site are expected from modeling studies. For example, Nef Lys82 is well positioned to interact with Glu99 of Hck, Glu98 of Fyn or Src, and Asp98 of Lck. Unlike Asp100-Arg77, the interaction of Nef Lys82 and Fyn Glu98 is not observed in the crystal structures of the Nef-Fyn SH3 complexes, probably as a result of these structures being derived from crystals grown in high concentrations of salt $(1 \mathrm{M})$. Modeling studies also suggest that both Hck and Lck can invoke the interaction of Glu118 from the SH3 domain with Nef Leu76.

Performing ITC experiments at high salt lowers the ionization strength of the interacting residues, reducing the potential for salt bridge formation. Table 1 shows that in both Hck and Src $0.5 \mathrm{M}$ salt has a negligible effect on affinity compared to the data in $0.15 \mathrm{M}$ salt. However, in both the loss of the salt bridging interaction appears to give rise to an increase in enthalpy and entropy compared to the interaction in $0.15 \mathrm{M}$ salt (for Hck SH3, $\Delta \Delta H_{0.5-0.15}=13.8$ $\mathrm{kJ} \cdot \mathrm{mol}^{-1} ; T \Delta \Delta S_{0.5-0.15}=13.7 \mathrm{~kJ} \cdot \mathrm{mol}^{-1}$; for Src SH3, $\left.\Delta \Delta H_{0.5-0.15}=3.1 \mathrm{~kJ} \cdot \mathrm{mol}^{-1} ; T \Delta \Delta S_{0.5-0.15}=4.4 \mathrm{~kJ} \cdot \mathrm{mol}^{-1}\right)$. These data are consistent with the removal of salt bridges giving rise to an increase in the degrees of freedom of the side chains (conformational entropy) which is almost exactly compensated for by the enthalpic loss of noncovalent interactions. Since the Asp100-Arg77 interaction prevails in the crystal structures solved in conditions of high salt, the thermodynamic data most likely reflect the removal of the additional salt bridging interactions outlined above. The difference in the $\Delta H$ and $T \Delta S$ between Hck and Src under the different salt conditions could be the result of the additional interaction that Hck can make involving Glu118 and the observation that Hck Glu99 is better positioned to interact with Nef Lys82 than Src Glu98.

The salt bridges that form in the complex structures appear to make a negligible contribution to the free energy of binding, but are probably important in locating and orientating the proteins prior to association. Indeed, on looking at the binding surface of the $\mathrm{SH} 3$ domains (Figure 5), there is little to distinguish them from one another for an incoming Nef molecule. Topologically, the SH3 binding surfaces are very similar, and the hydrophobic proline-rich binding sites 
offer little in terms of discrimination. However, the distribution of charged and polar groups in the RT loop region clearly shows some major points of possible recognition. Interestingly, looking at the two tightest binding SH3 domain surfaces, it becomes apparent that both Hck and Lck have similar charge distribution (both lacking the basic Arg96).

Thermodynamics of Binding of Full-Length Nef to Src Family SH3 Domains. As described above, structural information on Nef complexes to date has been derived from a deleted Nef construct. NMR data on full-length Nef have suggested that the $\mathrm{N}$-terminal is largely disordered (50). To investigate the effects on binding of the $\mathrm{N}$-terminal residues, ITC studies of the interaction of full-length Nef and Src family SH3 domains were performed. In the interaction with Hck and Src SH3 domains, the full-length Nef and the deleted Nef show very little difference in affinity (Table 1). The $K_{\mathrm{D}}$ reported for the Hck SH3-Nef interaction $(0.56 \mu \mathrm{M})$ is weaker than that previously reported $[0.19 \mu \mathrm{M} ;(10)]$. This is likely to be the result of differences in the Nef proteins used in the ITC studies. In our data, the HIV-1 Nef $\mathrm{LA}_{\mathrm{L}}$ (laboratory-adapted strain) variant is used which has Thr in the 71 position (as described in 19). In the large majority of more recently isolated HIV-1 strains, and in the HIV-1 $\mathrm{Nef}_{\mathrm{NL} 4-3}$ Thr71Arg construct used by Lee et al. (16), the Thr is replaced by an Arg. Arg71 can make an additional hydrogen bonding interaction on binding to $\mathrm{SH} 3$ domains (18) which the Thr71 form cannot (19). This may suggest that the HIV-1 virus has evolved to bind more tightly to SH3 domains.

The ITC binding data (Table 1) show that the interactions with the SH3 domains from Hck and Src are all highly enthalpy driven and a large entropic penalty is paid in each case. These $\Delta H$ and $T \Delta S$ data are very different from those obtained for the $\mathrm{Nef}_{\Delta 1-57}$ interactions. This is consistent with the reduction in conformational entropy and the increase in noncovalent bond formation resulting from folding of the $\mathrm{N}$-terminal going to the complex state. It is intriguing that, despite this possible gross conformational rearrangement, the affinity is similar for both full-length and deleted protein.

Summary of the Structural and Thermodynamic Basis of Specificity in Nef-SH3 Domain Binding. The interaction of $\mathrm{SH} 3$ domains with proline-rich sequences in ligands has been demonstrated to be an essential platform for binding (16); however, little specificity is offered by these sequences, and studies adopting peptides as mimics of these interactions suggest that affinities are low. Thus, both specificity and increased affinity are likely to be derived from elsewhere in the binding interface. Previous studies had implicated the key role of the interactions involving the RT loop of the SH3 domain $(16,53)$. In this study, we have investigated the interaction of four SH3 domains with the protein HIV-1 Nef. X-ray crystal structures and homology modeling show that for this binding to occur the RT loop of the SH3 domain has to be highly flexible to form an intimate interaction with the Nef binding site and several side chains undergo severe reorientation from their observed positions in the unbound $\mathrm{SH} 3$ structures. The SH3 domain of Hck is found to bind an order of magnitude more tightly to Nef than its Src family counterparts. The main variation in amino acids involved in binding between these domains occurs in the RT loop region; however, these changes do not seem to improve affinity by resulting in a large increase in specific interac- tions. For example, it is hard to see how the substitution of Ile96 to Arg between Hck and Fyn, respectively, can result in a decrease in binding of an order of magnitude, since in the crystal structure of the Fyn SH3-Nef complex the guanidinium group of the Arg is able to make hydrogen bonds with the Nef residues at the base of a deep pocket, and the incorporation of Ile introduces a water molecule into the pocket, presumably incurring a significant entropic cost (19). However, based on mutational studies in Fyn, the presence of the Ile96 residue in the binding interface is important for high-affinity binding (16).

In the tightest binding interaction between Hck SH3 and Nef, the residues in the RT loop of the unbound peptide are highly flexible compared to those of other SH3 domains. In the RT loop of Hck, Ile96 and His97 replace Arg and Thr, respectively, in Fyn and Src. These substitutions preclude a hydrogen bonded network forming, leading to a greater pliancy. Thus, these residues can facilitate the interaction with the Nef binding site; since the RT loop is already mobile, the breaking of hydrogen bonds is not required to make the interface with the Nef molecule. This is emphasized by the fact that the substitution of both Ile and His into positions 96 and 97 in Fyn gives rise to a higher affinity interaction (16).

It should be emphasized that the recently solved structures of two proteins from the Src family of tyrosine kinases (24, 25 ) show that in the 'closed' form the $\mathrm{SH} 3$ domain interacts with a loop region linking the N-lobe of the kinase to the SH2 domain. The residues in this loop in the 'closed' form adopt a PPII-like helix. Thus, it is possible that the Nef protein has to compete for this site with this intramolecular interaction. Furthermore, although this intramolecular interaction is less than optimal and hence weak, since the sequence of the interacting loop shows some variability between the various members of the Src family, some additional level of specificity could be invoked.

Comparison of the SH3 domains reveals the binding of Hck represents an intriguing variation on the 'induced fit' mechanism of binding since although the RT loop of the high-affinity SH3 ligand is freely available other SH3 domains require the expenditure of additional enthalpy to break hydrogen bonds to allow this to occur [analogous to that hypothesized for vascular endothelial growth factor (54)]. This results in an additional level of specificity in $\mathrm{SH} 3$ domain interactions providing a further challenge in the identification of pharmaceutical inhibitors.

\section{ACKNOWLEDGMENT}

We express our thanks to Drs. S. Benichou for the Hck SH3 domain expression vector, H. Dutarte for the Lck SH3 domain expression vector, C. Le Grimellec for access to ITC, L. Chiche for assistance with modeling studies, and M. Roth and R. Kahn for their help using the D2AM ESRF beamline. We express our gratitude to Dr. D. A. Renzoni for comments on the manuscript and assistance with the figures.

\section{REFERENCES}

1. Cicchetti, P., Mayer, B. J., Thiel, G., and Baltimore, D. (1992) Science 257, 803-806.

2. Ren, R., Mayer, B. J., Cicchetti, P., and Baltimore, D. (1993) Science 259, 1157-1161. 
3. Kuriyan, J., and Cowburn, D. (1993) Curr. Opin. Struct. Biol. 3, 828-837.

4. Musacchio, A., Saraste, M., and Wilmanns, M. (1994) Nat. Struct. Biol. 1, 546-551.

5. Feng, S., Chen, J. K., Yu, H., Simon, J. A., and Schreiber, S. L. (1994) Science 266, 1241-1247.

6. Goudreau, N., Cornille, F., Duchesne, M., Parker, F., Tocque, B., Garbay, C., and Roques, B. P. (1994) Nat. Struct. Biol. 1, 898-907.

7. Lim, W. A., Richards, F. M., and Fox, R. O. (1994) Nature $372,375-379$.

8. Terasawa, H., Kohda, D., Hatanaka, H., Tsuchiya, S., Ogura, K., Nagata, K., Ishii, S., Mandiyan, V., Ullrich, A., Schlessinger, J., and Inagaki, F. (1994) Nat. Struct. Biol. 1, 891-897.

9. Yu, H., Chen, J. K., Feng, S., Dalgarno, D. C., Brauer, A. W., and Schreiber, S. L. (1994) Cell 76, 933-945.

10. Lim, W. A., and Richards, F. M. (1994) Nat. Struct. Biol. 1, $221-225$.

11. Sparks, A. B., Quilliam, L. A., Thorn, J. M., Der, C. J., and Kay, B. K. (1994) J. Biol. Chem. 269, 23853-23856.

12. Viguera, A. R., Martinez, J. C., Filmanov, V. V., Mateo, P. L., and Serrano, L. (1994) Biochemistry 33, 2142-2150.

13. Rickles, R., Botfield, M. C., Weng, Z., Taylor, J., Green, O. M., Brugge, J., and Zoller (1994) EMBO J. 13, 5598-5604.

14. Rickles, R. J., Botfield, M. C., Zhou, X.-M., Henry, P. A., Brugge, J., and Zoller, M. J. (1995) Proc. Natl. Acad. Sci. U.S.A. 92, 10909-10913.

15. Alexandropoulos, K., Cheng, G., and Baltimore, D. (1995) Proc. Natl. Acad. Sci. U.S.A. 92, 3110-3114.

16. Lee, C.-H., Leung, B., Lemmon, M. A., Zheng, J., Cowburn, D., Kuriyan, J., and Saksela, K. (1995) EMBO J. 14, 50065015.

17. Renzoni, D., Pugh, D. J. R., Siligardi, G., Das, P., Morton, C. J., Rossi, C., Waterfield, M. D., Campbell, I. D., and Ladbury, J. E. (1996) Biochemistry 35, 15646-15653.

18. Lee, C.-H., Saksela, K., Mirza, U. A., Chait, B. T., and Kuriyan, J. (1996) Cell 85, 931-942.

19. Arold, S., Franken, P., Strub, M.-P., Hoh, F., Benichou, S., Benarous, R., and Dumas, C. (1997) Structure 5, 1361-1372.

20. Noble, M. E., Musacchio, A., Saraste, M., Courtneidge, S. A., and Weirenga, R. K. (1993) EMBO J. 12, 2617-2624.

21. Morton, C. J., Pugh, D. J. R., Brown, E. L. J., Kahman, J. D., Renzoni, D. A. C., and Campbell, I. D. (1996) Structure 4, $705-714$

22. Yu, H., Rosen, M. K., Shin, T. B., Seidel-Dugan, C., Brugge, J. S., and Schreiber, S. L. (1992) Science 258, 1665-1668.

23. Eck, M. J., Atwell, S. K., Shoelson, S. E., and Harrison, S. C. (1994) Nature 268, 764-769.

24. Xu, W., Harrison, S. C., and Eck, M. J. (1997) Nature 385, 595-602.

25. Sicheri, F., Moareffi, I., and Kuriyan, J. (1997) Nature 385, 602-609.

26. Saksela, K., Cheng, G., and Baltimore, D. (1995) EMBO J. 14, 484-491.

27. Collette, Y., Dutartre, H., Benziane, A., Ramos-Morales, F., Benarous, R., Harris, M., and Olive, D. J. (1996) Biol. Chem.
$271,6333-6341$.

28. Greenway, A., Azad, A., Mills, J., and McPhee, D. (1996) J. Virol. 70, 6701-6708.

29. Saksella, K. (1997) Front. Biosci. 2, 606-618.

30. Trono, D. (1995) Cell 82, 189-192.

31. Schwartz O., Marechal, V., Le Gall, S., Lemonnier, F., and Heard, J. M. (1996) Nat. Med. 2, 388-342.

32. Collins, K. L., Chen, B. K., Kalams, S. A., Walker, D. W., and Baltimore, D. (1998) Nature 391, 397-401.

33. Briggs, S. D., Sharkey, M., Stevenson, M., and Smithgall, T. E. (1997) J. Biol. Chem. 272, 17899-17902.

34. Iafrate, J., Bronson, S., and Skowronski, J. (1997) EMBO J. $16,673-684$.

35. Littman, D. R. (1994) Curr. Biol. 4, 618-620.

36. Gratton, S., Yao, X. J., Venkatescan, S., Cohen, E. A., and Sekalay, R. P. (1996) J. Immunol. 8, 3305-3311.

37. Salghetti, S., Mariani, R., and Skowronski, J. (1995) Proc. Natl. Acad. Sci. U.S.A. 92, 349-353.

38. Lang, S. M., Iafrate, J. A., Stahl-Hennig, C., Kuhn, E. M., Nisslein, T., Kaup, Haupt F.-J., Hunsmann, M., Skowronski, J., and Kirchhoff, F. (1997) Nat. Med. 3, 860-865.

39. Kabsch, W. (1993) J. Appl. Crystallogr. 26, 795-800.

40. Navaza, J. (1994) Acta Crystallogr. A 50, 157-163.

41. Brünger, A. T. (1992) X-PLOR Manual, Version 3.1. Yale University, New Haven, CT.

42. Jones, T. A., Zou, J. Y., Cowan, S. W., and Kjeldgaard, M. (1993) Acta Crystallogr. D 49, 148-157.

43. Laskowski, R. A., MacArthur, M. W., Moss, D. S., and Thornton, J. M. (1993) J. Appl. Crystallogr. 26, 283-291.

44. Vriend, G. (1990) J. Mol. Graph. 8, 52-56.

45. Wiseman, T., Williston, S., Brandts, J. F., and Lin, L.-N. (1989) Anal. Biochem. 179, 131-137.

46. Ladbury, J. E., and Chowdhry, B. Z. (1996) Chem. Biol.

47. Ladbury, J. E., Lemmon, M. A., Zhou, M., Green, J., Botfield, M. C., and Schlessinger, J. (1995) Proc. Natl. Acad. Sci. U.S.A. 92, 3199-3203.

48. Tuffery, P., Etchbest, C., Hazeout, S., and Lavery, R. (1991) J. Biomol. Struct. Dyn. 8, 1267-1289.

49. Pearlman, D. A., Case, D. A., Cadwell, J. W., Ross, W. S., Chaetham, T. E., Ferguson, D. M., Seibel, G. L., Singh, U. C., Weiner, P. K., and Kollman, P. A. (1995) Comput. Phys. Commun. 91, 1-41.

50. Grzesiak, S., Bax, A., Hu, J. S., Kaufman, J., Palmer, I., Stahl, S. J., Tjandra, N., and Wingfield, P. T. (1997) Protein Sci. 6, $1248-1263$.

51. Ladbury, J. E. (1996) Chem. Biol. 3, 973-980.

52. Engen, J. R., Smithgall, T. E., Gmeiner, W. H., and Smith, D. L. (1997) Biochemistry 36, 14384-14391.

53. Erpel, T., Superti-Furga, G., and Courtneidge, S. A. (1995) EMBO J. 14, 963-975.

54. Muller, Y. A., Christinger, H. W., Keyt, B. A., and de Vos, A. M. (1997) Structure 5, 1325-1338.

BI980989Q 